\title{
Análisis de la influencia del estado superficial en la energía absorbida en la interacción láser $\mathrm{CO}_{2}$ - aluminio
}

\author{
J. Oñoro*, R. Bermejo* y L. Sánchez-Ibarzabal*
}

\begin{abstract}
Resumen En este trabajo se han realizado ensayos de refusión, en dos aleaciones de aluminio, de los grupos Al-Mg-Si y Al-Zn-Mg, con diferentes tratamientos superficiales. La aleación del grupo Al-Mg-Si ha presentado una mayor absorción de energía y un mejor acabado superficial. Los tratamientos superficiales con pinturas, han conducido a un ligero aumento de la energía absorbida, pero han producido un aumento considerable de la porosidad. El aumento de la rugosidad superficial ha favorecido el aumento de la absorción de energía en las aleaciones estudiadas. El tratamiento químico previo a los ensayos de refusión ha conducido a un aumento de la penetración y de la energía absorbida, especialmente en la aleación del grupo Al-Mg-Si.
\end{abstract}

Palabras clave Láser de $\mathrm{CO}_{2}$. Aluminio. Energía absorbida. Superficie.

\section{Surface influence of energy absorbed in $\mathrm{CO}_{2}$ laser-aluminium interaction}

\begin{abstract}
In this work bead-on-plates tests have been performed with $\mathrm{CO}_{2}$ laser beam, on $\mathrm{Al}-\mathrm{Mg}-\mathrm{Si}$ and $\mathrm{Al}-\mathrm{Zn}-\mathrm{Mg}$ aluminium alloys with different surface treatments. Al-Mg-Si alloy has presented more energy absorbed and best surface finish. The surface treatment with paints has produced an increase of absorbed energy, but with an important porosity in the fusion zone. The increase of surface rugosity has produced an increase of absorbed energy. The chemical treatments previous at bead-on-plates test have produced an important increasing in absorbed energy, especially in Al-Mg-Si alloy.
\end{abstract}

Keywords $\quad \mathrm{CO}_{2}$ laser. Aluminium. Absorbed energy. Surface.

\section{INTRODUCCIÓN}

El haz láser de $\mathrm{CO}_{2}$ es un rayo de luz coherente y monocromático de alta densidad de energía, es decir, una radiación fotónica concentrada de una longitud de onda de $10,6 \mu \mathrm{m}$, que se encuentra dentro del campo infrarrojo. La tecnología del tratamiento de materiales con láser de $\mathrm{CO}_{2}$ se basa en la concentración de este rayo de luz sobre la superficie del material a tratar, donde se transforma la energía fotónica en energía térmica, produciendo un aumento en la temperatura de la superficie del material. En general, la cantidad de energía absorbida por los materiales es baja, al producirse notables pérdidas por reflexión del haz ${ }^{[1-3]}$, especialmente si la energía aportada es de baja densidad. $\mathrm{Si}$, por el contrario, la densidad de energía es alta (igual o superiores a $10^{5} \mathrm{~W} / \mathrm{cm}^{2}$ ), se produce una profunda penetración del láser dentro del metal, formando una columna llena de un gas metálico ionizado (plasma), cuya energía térmica se transfiere a la zona metálica circundante.

El aluminio y sus aleaciones son materiales difíciles de fundir con láser. El pobre acoplamiento del haz sobre la superficie del aluminio es debido en parte a la alta densidad de electrones libres en el sólido (responsables de su alta conductividad térmica y eléctrica), haciendo del aluminio uno de los mejores reflectores de la luz ${ }^{[4]}$. A este efecto hay que añadirle la formación de un plasma bloqueante del haz cuando en la composición de la aleación de aluminio existen elementos, tales como el magnesio y cinc, de baja temperatura de vaporización ${ }^{[5]}$.

(*) Departamento Ingeniería y Ciencia de los Materiales. ETSI Industriales. UPM, C/ José Gutiérrez Abascal, 2. 28006 Madrid, España. E-mail: onoro@materials.upm.es 
La energía asociada a un fotón de un láser de $\mathrm{CO}_{2}$ es de 0,1 ev, baja en comparación con la de un electrón de un haz de electrones, del orden de 100.000 ev. Por esta razón, el haz láser tiene una gran tendencia a interaccionar con los electrones libres existentes en el plasma (gas de protección y vapor metálico formado por la incidencia del haz sobre el aluminio). Este plasma, en el caso del aluminio, tiende a dispersar parte del haz (efecto desfocalizador), dando lugar a una interacción inestable con zonas fundidas anchas y poco profundas, comparadas con las obtenidas con otros materiales, y un aspecto superficial irregular ${ }^{[2]}$.

La cantidad de energía absorbida en la interacción láser-aluminio depende de una gran cantidad de factores, entre ellos: el tipo de aleación, la geometría de la pieza, el acabado superficial, la potencia del haz, la geometría del haz, la naturaleza del gas de protección, la focalización, etc. ${ }^{[1-3]}$. La modificación de estos parámetros produce importantes variaciones en las condiciones de transferencia energética entre el haz láser y el material sobre el que incide, lo que supone, por un lado, una gran dificultad para encontrar los parámetros idóneos necesarios para cada aplicación, pero, por otro, permite una gran versatilidad en las interacciones láser-materia, permitiendo, con pequeñas modificaciones de dichos parámetros, llevar a cabo diferentes procesos como pueden ser corte, soldadura o tratamientos térmicos superficiales $\left.{ }^{[6 \mathrm{y}} 7\right]$.

En los estudios de la interacción láser-aluminio, la realización de ensayos de refusión, es una herramienta ampliamente utilizada para el análisis de las condiciones de ensayo ${ }^{[4}$ y 5$]$. Sin embargo, la evaluación de los resultados obtenidos, es generalmente cualitativa: función de la geometría, el aspecto superficial, etc., de los ensayos realizados. En este trabajo se han realizado ensayos de refusión en dos aleaciones de aluminio de los grupos Al-Mg-Si (6082) y Al-Zn-Mg (7020), con diferentes tratamientos superficiales, que suponen una importante modificación de las características superficiales de las aleaciones estudiadas. La evaluación de la naturaleza de la interacción láser-aluminio para cada aleación y cada tratamiento superficial se ha realizado cuantificando los resultados de los ensayos de refusión por la energía absorbida en cada ensayo utilizando una modelización, presentada en una publicación anterior ${ }^{[8]}$, que permite una cuantificación de dicha energía a partir de valores geométricos obtenidos directamente de los ensayos y las constantes físicas de las aleaciones estudiadas.

\section{MATERIALES Y PROCEDIMIENTO EXPERIMEN- TAL}

Las muestras para la realización de los ensayos han sido probetas de las aleaciones 6082 y 7020 de dimensiones $200 \times 200 \times 4 \mathrm{~mm}$, la composición química de las mismas se ha recogido en la tabla I.

Las muestras han sido tratadas superficialmente para obtener los siguientes acabados:

- Lijadas con granulometría 100, 320 y 600.

- Atacada superficialmente con solución acuosa $50 \mathrm{gr} / \mathrm{l} \mathrm{NaOH}$.

- Atacada superficialmente con solución acuosa $100 \mathrm{gr} / 1 \mathrm{~K}_{2} \mathrm{CO}_{3}$.

- Atacada superficialmente con solución acuosa $110 \mathrm{gr} / 1 \mathrm{Na}_{2} \mathrm{CO}_{3}$.

- Pintadas con pigmento de grafito y con pintura epoxi negra.

Los parámetros del haz láser utilizados en todos los ensayos de refusión han sido idénticos para ambos materiales y dichos datos se han recogido en la tabla II. La potencia real suministrada por el haz láser se ha determinado experimentalmente a través de un calorímetro instalado a la salida del láser. Los ensayos de refusión producen una fusión parcial del material ensayado; los parámetros de estos ensayos han sido elegidos para alcanzar una fusión suficiente del material sin que se llegara a perforar. Esto ha permitido disponer en todos los ensayos de unas condiciones físicas de transferencia de calor muy similares.

Una vez realizados los ensayos de refusión, se procedió a seccionar las muestras en su parte

Tabla I. Composición química de las aleaciones estudiadas

Table I. Chemical composition of alloys analysed

\begin{tabular}{lccccccccc}
\hline Aleación & $\mathrm{Si}$ & $\mathrm{Fe}$ & $\mathrm{Cu}$ & $\mathrm{Mn}$ & $\mathrm{Mg}$ & $\mathrm{Cr}$ & $\mathrm{Zn}$ & $\mathrm{Ti}$ & $\mathrm{Al}$ \\
\hline 6082 & 1,05 & 0,5 & 0,1 & 0,7 & 0,9 & 0,25 & 0,2 & 0,1 & 96,2 \\
7020 & 0,35 & 0,4 & 0,2 & 0,3 & 1,2 & 0,25 & 4,5 & 0,15 & 92,7 \\
\hline
\end{tabular}


Análisis de la influencia del estado superficial en la energía absorbida en la interacción láser $\mathrm{CO}_{2}$ - aluminio

J. OÑORO, R. BERMEJO Y L. SÁNCHEZ-IBARZABAL

Tabla II. Parámetros del haz láser utilizados en los ensayos de refusión

Table II. Laser beam parameters used on bead-on-plates testing

\begin{tabular}{lc}
\hline Equipo LASER & RS-6000 \\
\hline Modo & TEM 00 \\
Energía Nominal $(\mathrm{W})$ & 2000 \\
Energía Incidente $(\mathrm{W})$ & 1700 \\
Longitud Focal $(\mathrm{mm})$ & 150 \\
Punto Focal $(\mathrm{mm})$ & -1 \\
Boquilla-Pieza $(\mathrm{mm})$ & 5 \\
Gas de Protección & $\mathrm{Ar}$ \\
Caudal de Gas $(1 / \mathrm{min})$ & 15 \\
Velocidad de Soldeo $(\mathrm{m} / \mathrm{min})$ & 1 \\
\hline
\end{tabular}

central y se prepararon las secciones metalográficamente. Este análisis ha permitido determinar tanto la geometría de la zona fundida como la calidad metalúrgica de la misma asociada a cada tratamiento. La profundidad de la zona fundida se determinó en un proyector de perfiles y el área fundida midiendo la superficie por análisis de imagen. Los parámetros y unidades utilizados en la cuantificación de la energía absorbida se han recogido en la tabla III. Los valores de las constantes físicas y de los parámetros utilizados en la modelización, se han recogido en la tabla IV.

\section{ANÁLISIS DE RESULTADOS Y DISCUSIÓN}

Los resultados obtenidos en los ensayos de refusión se han recogido en la tabla $\mathrm{V}$, conjuntamente con los valores de energía absorbida obtenidos para cada aleación y cada tratamiento superficial.

Los valores de la energía absorbida se han calculado a partir de dos ecuaciones que calculan la energía transmitida por conducción y la absorbida por fusión y vaporización que son, respectivamente:

$$
E_{c}=\frac{\left(T_{m}-T_{0}\right) e \pi \rho C\left(Z_{m}+Z_{0}\right)^{2}}{2} \mathrm{v}
$$

$$
E_{m V}=\left(A_{m} L_{m}+A_{V} L_{V}\right) v
$$

donde, los valores de dichas ecuaciones son propiedades físicas o parámetros experimentales que se pueden obtener de los ensayos de refusión realizados $\left[Z_{0}=\left(4-Z_{m}\right)\left(Z_{m} / 4\right)^{2} ; A_{V}=r_{B} 3 Z_{m} / 2\right]$. En la figura 1 se indican los valores de los parámetros geométricos utilizados en la modelización. Una descripción más exhaustiva del modelo utilizado puede verse en trabajos publicados ${ }^{[8]}$.

Tabla III. Parámetros y unidades utilizados en la modelización de la energía absorbida

\begin{tabular}{|c|c|}
\hline PARÁMETROS & UNIDADES \\
\hline$E_{C}:$ Energía absorbida por unidad de tiempo & W \\
\hline$E_{m v}$ : Energía absorbida en la fusión y vaporización & W \\
\hline $\mathrm{T}_{0}:$ Temperatura inicial del material & $\mathrm{K}$ \\
\hline$T_{m}:$ Temperatura de fusión del material & $\mathrm{K}$ \\
\hline v: Velocidad de desplazamiento del haz láser & $\mathrm{m} / \mathrm{s}$ \\
\hline $\mathrm{r}_{\mathrm{B}}$ : Radio del haz láser & $\mathrm{m}$ \\
\hline$Z_{0}:$ Profundidad a la que el calor afecta durante la interacción del haz & $\mathrm{m}$ \\
\hline $\mathrm{Z}_{\mathrm{m}}:$ Profundidad de la zona fundida & $\mathrm{m}$ \\
\hline$Z_{\mathrm{t}}:$ Espesor del material & $\mathrm{m}$ \\
\hline ?: Densidad & $\mathrm{Kg} / \mathrm{m}^{3}$ \\
\hline C: Calor específico & $\mathrm{J} / \mathrm{KgK}$ \\
\hline$A_{m}:$ Area fundida & $m^{2}$ \\
\hline$A_{v}:$ Area vaporizada & $\mathrm{m}^{2}$ \\
\hline $\mathrm{L}_{\mathrm{m}}:$ Calor latente de fusión & $\mathrm{J} / \mathrm{Kg}$ \\
\hline $\mathrm{L}_{\mathrm{v}}:$ Calor latente de vaporización & $\mathrm{J} / \mathrm{Kg}$ \\
\hline e: Base de los logaritmos neperianos $=2,718$ & \\
\hline
\end{tabular}

Table III. Parameters and units used in the absorbed energy model 
Tabla IV. Propiedades físicas y parámetros experimentales

Table IV. Physical properties and experimental parameters

\begin{tabular}{lcc}
\hline ALEACIÓN & 6082 & 7020 \\
\hline$\rho\left(\mathrm{Kg} / \mathrm{dm}^{3}\right)$ & 2,7 & 2,77 \\
$T_{\mathrm{m}}\left({ }^{\circ} \mathrm{C}\right)$ & 655 & 650 \\
$\mathrm{C}(\mathrm{J} / \mathrm{KgK})$ & 896 & 875 \\
$\mathrm{~L}_{\mathrm{m}}(\mathrm{KJ} / \mathrm{Kg})$ & 386 & 384 \\
$\mathrm{~L}_{\mathrm{V}}(\mathrm{KJ} / \mathrm{Kg})$ & 11.000 & 11.000 \\
$V(\mathrm{~m} / \mathrm{min})$ & 1 & 1 \\
$\mathrm{~T}_{0}\left({ }^{\circ} \mathrm{C}\right)$ & 20 & 20 \\
$\mathrm{Z}_{0}(\mathrm{~mm})$ & 4 & 4 \\
$\mathrm{r}_{B}(\mathrm{~mm})$ & 0,25 & 0,25 \\
$E_{\mathrm{i}}(\mathrm{W})$ & 1.700 & 1.700 \\
\hline
\end{tabular}

Los tratamientos superficiales realizados han supuesto una importante modificación de las características superficiales de las aleaciones tratadas. Estos tratamientos pretenden cambiar la naturaleza de las superficies de manera que se consiga una modificación de las condiciones de interacción del haz láser con la superficie de aluminio, para hacerla menos reflectante al haz y por tanto con mayor capacidad absorbente de la energía aplicada.

Los tratamientos realizados con lijas de diferente granulometría han conducido en ambas aleaciones a que cuanto más rugoso ha sido el acabado, se ha producido una mayor absorción de energía. Las superficies, al tener una mayor rugosidad, provocan un mayor número de reflexiones secundarias que tienen su origen en las paredes de las ondulaciones.

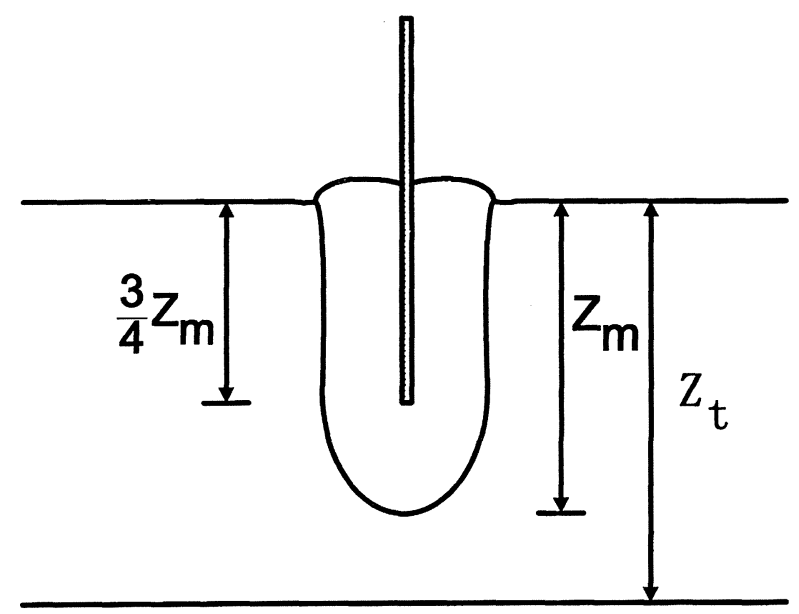

Figura 1. Geometría de las zonas fundidas y vaporizadas en relación con el espesor de la pieza.

Figure 1. Melted and vaporized areas geometry in relation to the piece thickness.

Los rayos reflejados por dichas paredes, causan una absorción estimulada cuando el haz incidente interfiere con los rayos reflejados, conduciendo en definitiva a una mayor absorción de energía ${ }^{[9]}$.

Los tratamientos de ataque químico superficial tenían por objeto reducir la reflexión, por la formación de capas de aluminatos que producen un ennegrecimiento superficial de las aleaciones de aluminio. Estos tratamientos son los que han conseguido en la aleación 6082 una mayor absorción de energía, especialmente el realizado con $\mathrm{Na}_{2} \mathrm{CO}_{3}$, al haber conducido a una importante reducción de la reflexión del haz láser en el aluminio. El efecto de estos tratamientos sobre la

Tabla V. Valores de geométricos de la zona fundida y energía absorbida, obtenidos en los ensayos de refusión, en las aleaciones de aluminio 6082 y 7020

Table V. Energy absorbed and bead-on-plate geometric results in the fusion zone, obtained in 6082 and 7020 aluminium alloys

\begin{tabular}{|c|c|c|c|c|c|c|}
\hline \multirow[t]{2}{*}{$\begin{array}{l}\text { PREPARACIÓN } \\
\text { SUPERFICIAL }\end{array}$} & \multicolumn{2}{|c|}{$\begin{array}{l}\text { PROFUNDIDAD } \\
Z_{m}(\mathrm{~mm})\end{array}$} & \multicolumn{2}{|c|}{$\begin{array}{c}\text { AREA } \\
A_{m}\left(\mathrm{~mm}^{2}\right)\end{array}$} & \multicolumn{2}{|c|}{$\begin{array}{l}\text { ENERGÍA ABSORBIDA } \\
\qquad E_{c}+E_{m V}(W)\end{array}$} \\
\hline & 6082 & 7020 & 6082 & 7020 & 6082 & 7020 \\
\hline Lija 100 & 1,98 & 1,80 & 3,76 & 2,65 & 918,72 & 766,29 \\
\hline Lija 320 & 1,64 & 1,94 & 2,54 & 2,48 & 649,82 & 720,23 \\
\hline Lija 600 & 1,54 & 1,50 & 2,46 & 1,96 & 582,24 & 550,11 \\
\hline $\mathrm{NaOH}$ & 2,57 & 1,75 & 4,83 & 2,28 & $1.414,29$ & 723,79 \\
\hline $\mathrm{K}_{2} \mathrm{CO}_{3}$ & 2,60 & 1,80 & 4,22 & 2,40 & $1.428,13$ & 761,86 \\
\hline $\mathrm{Na}_{2} \mathrm{CO}_{3}$ & 2,64 & 1,67 & 4,83 & 2,30 & $1.471,18$ & 668,17 \\
\hline P. Grafito & 2,11 & 1,98 & 4,14 & 2,85 & $1.027,32$ & 904,68 \\
\hline P. Epoxi & 1,30 & 1,85 & 1,80 & 2,84 & 504,64 & 806,30 \\
\hline
\end{tabular}


aleación 7020 no han sido tan marcados y podemos decir que no se ha producido una diferencia significativa en relación a los tratamientos de lijado.

El tratamiento químico de aleaciones Al-Mg-Si para la formación de capas menos reflectoras a la acción del láser de $\mathrm{CO}_{2}$, puede ser un tratamiento previo a un proceso de soldadura con láser de importante utilidad para aumentar la eficiencia del mismo. Con estos tratamientos se ha conseguido aumentar la absorción de energía al doble de lo obtenido, en las mismas condiciones, con las probetas únicamente lijadas. Esta absorción de energía ha dado lugar, tanto a una mayor penetración de la zona fundida como a un mayor tamaño de la misma. El haber conseguido con este tratamiento un aumento de penetración en unos valores en torno al $60 \%$ indica que estos tratamientos pueden tener una aplicación práctica importante para la mejora y el aumento del rendimiento en la realización de uniones soldadas con estas aleaciones. La zona fundida en todas las muestras tratadas químicamente se han visto libres de porosidad en inclusiones, como puede apreciarse en la figura 2.

Los dos recubrimientos de pintura han mostrado un diferente comportamiento frente a la interacción con el haz láser. En la aleación 6082 ha sido donde la diferencia de absorción de energía ha sido más marcada aunque también ha sido relevante en la 7020. En esta última, el tratamiento superficial con pinturas es el que ha conducido a una mayor energía absorbida. La razón de este diferente comportamiento parece deberse a la mayor rugosidad superficial de la pintura con pigmento de grafito, que presentaba un aspecto granuloso y rugoso, donde se apreciaban las líneas de aplicación

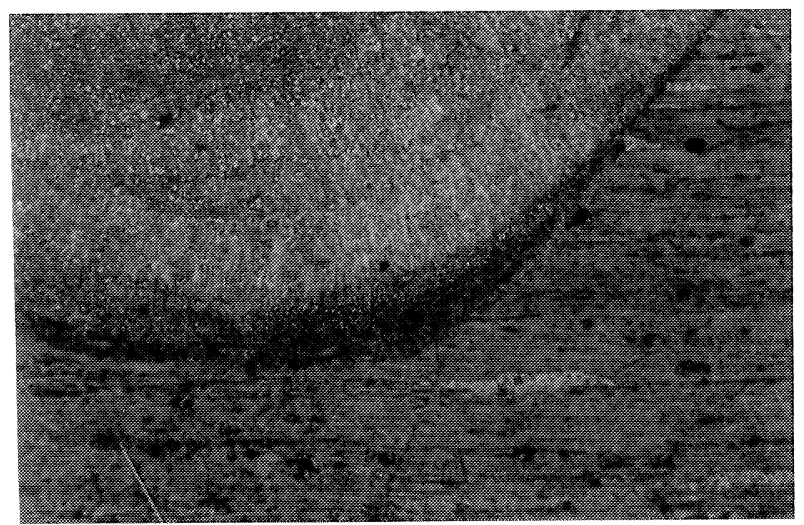

Figura 2. Aleación 7020. x100, ataque Keller. Muestra tratada superficialmente con $\mathrm{NaOH}$.

Figure 2. 7020 alloy. x100, Keller etching. Sample surface treated with $\mathrm{NaOH}$. de la pintura. Por el contrario, la pintura negra epoxi presentaba un aspecto superficial liso, brillante y satinado.

Los recubrimientos realizados con pinturas han presentado en todos los casos un alto nivel de porosidad e inclusiones, como puede apreciarse en la figura 3. La porosidad se ha debido mayoritariamente a la combustión de la película de pintura. Durante los ensayos de refusión se produjo un gran desprendimiento de humos, especialmente en el recubrimiento con resina epoxi.

La mayor absorción de energía que se ha producido en las superficies atacadas o pintadas, ha sido también causada porque la presencia de la fina capa orgánica o inorgánica, sobre la superficies metálicas, que ha reducido la densidad electrónica a nivel superficial, y por tanto, ha producido una reducción de las perdidas por reflexión, con el consiguiente aumento de la absorción.

De los resultados de absorción de energía expuestos en la tabla $\mathrm{V}$, podemos decir que la aleación 6082 ha presentado una mayor absorción de energía en la mayoría de las preparaciones superficiales realizadas. Estas diferencias se han producido, tanto por una mayor profundidad como por un área superior de la zona fundida. Si se tiene en cuenta que las diferencias entre las constantes físicas de ambos materiales, por si mismas, no conducen a valores de energía absorbida muy distintos, a igualdad de los parámetros experimentales. Este distinto resultado de energía absorbida es debido al mayor contenido de elementos de bajo punto de vaporización ( $\mathrm{Zn}$ y $\mathrm{Mg}$ ) en la aleación 7020 , que como se mencionó anteriormente, produce un plasma bloqueante del haz que reduce la absorción de la energía incidente.

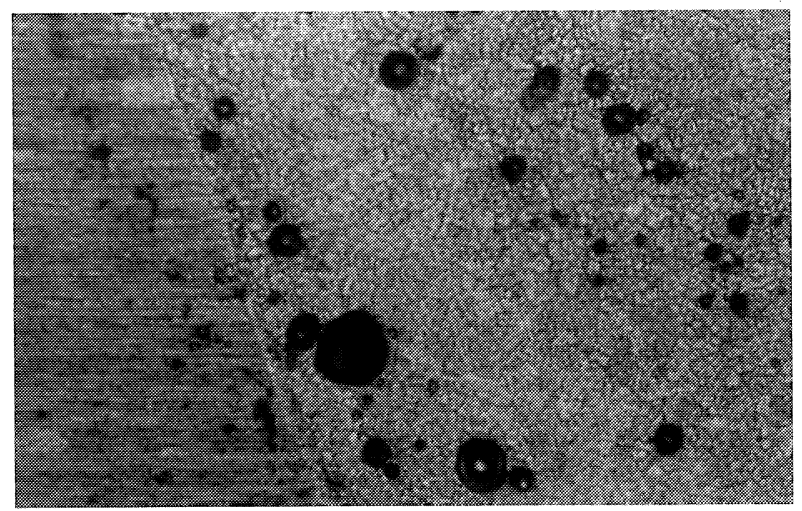

Figura 3. Aleación 7020. ×200, ataque Keller. Muestra pintada superficialmente con pintura epoxi negra.

Figure 3. 7020 alloy. x200, Keller etching. Sample surface painting with black epoxy paint.

Rev. Metal. Madrid Vol. Extr. (2005) 40-45 
El comportamiento de ambas aleaciones ha sido también distinto en cuanto al tipo de recubrimiento producido. La aleación 6082 ha presentado valores más elevados, en cuanto al tamaño del área fundida y energía absorbida, en las probetas atacadas superficialmente que en las lijadas y pintadas. La pintura a base de grafito ha ofrecido un resultado similar, aunque menos acusado.

\section{CONCLUSIONES}

- Se han realizado ensayos de refusión con láser de $\mathrm{CO}_{2}$ sobre muestras de aleaciones 6082 y 7020 , con diferentes tratamientos superficiales, analizándose la energía absorbida en la interacción láser-aluminio. Esta energía se ha cuantificado mediante un modelo que tiene en cuenta tanto el calor transmitido al resto de la pieza por conducción, como el necesario para la fusión y evaporación en la zona de formación del plasma, durante la aplicación del haz láser.

- Un aumento de la rugosidad superficial, en ambas aleaciones, ha conducido a una mayor $a b$ sorción de energía.

- La realización de un ataque químico superficial que produzca un ennegrecimiento por la presencia de aluminatos ha conducido a un aumento importante de la absorción de energía en la aleación 6082. Sin embargo, en la aleación 7020 su efecto ha sido poco significativo. La utilización de estos ataques superficiales en aleaciones $\mathrm{Al}-\mathrm{Mg}$-Si, antes de un proceso de soldadura con láser, parece una alternativa de gran interés tecnológico, para aumentar la penetración y rendimiento del proceso, manteniendo un buen aspecto superficial y una zona fundida sana.

- La aplicación de pinturas ha producido un resultado desigual. La pintura de grafito ha conducido a un aumento de la absorción de ener- gía; no así la pintura epoxi negra, especialmente en la aleación 6082 donde la absorción de energía ha sido muy baja. Por otro lado, el deficiente acabado superficial y la importante presencia de porosidad en la zona fundida, parecen hacer inviable su utilización práctica.

- La aleación 6082 ha sido la que ha presentado una mayor absorción de energía y un mejor acabado superficial, en la mayoría de los ensayos realizados. Esto ha sido debido a la menor presencia, en su composición, de elementos de bajo punto de fusión.

\section{Agradecimientos}

Los autores agradecen a la UPM la ayuda concedida a proyectos multidisciplinares de $\mathrm{I}+\mathrm{D}$, que ha hecho posible la realización de este trabajo.

\section{REFERENCIAS}

[1] W.M. StEen, Laser material processing. Springer-Verlag, London, 1991, pp. 12, 109, 114, 136.

[2] J. Mazumder, J. Met. 34 (1982) 16-24.

[3] D.T. Swift-Hook y A.E.F. Gick, Weld. Res. (1973) 492s499s.

[4] J.R. DAvis, Aluminum and Al alloys, Ed. ASM Int. Materials Park, OH, 1994, p. 644.

[5] C.A. Huntington y T.W. Eagar, Weld. Res. (1983) 105s$107 \mathrm{~s}$.

[6] G. Muñz, R. Conde, B.J. Fernández, R. Varela, I. García y J. de Damborenea, Rev. Metal. Madrid 39 (2003) 443-451.

[7] J.M. Amado, C. Álvarez, G. Nicolás, A.J. López, J.A. Pérez, A. Ramil, E. SaAvedra, J. Sanesteban, M.J. Tobar y A. YÁñeZ, Rev. Metal. Madrid 40 (2004) 365-368.

[8] J. Oñoro, R. Bermejo, A. Portolés y R. Gamboa, Rev. Metal. Madrid 34 (1998) 421-427.

[9] T.J. Wieting y J.L. Derosa, J. Appl. Phys. 50 (1979) 10711078. 